\title{
Necesidades de la familia y de la escuela en la educación de niños con discapacidad intelectual
}

School and family needs in the education of intellectual disability children 


\title{
Necesidades de la familia y de la escuela en la educación de niños con discapacidad intelectual ${ }^{1}$
}

\section{School and family needs in the education of intellectual disability children}

\author{
Lina María Acuña Arango ${ }^{2}$, Victoria Eugenia Cabrera García ${ }^{3}$, \\ Diana Carolina Medina Casallas ${ }^{4}$, Flor Alba Lizarazo Sandoval ${ }^{5}$ \\ Universidad de La Sabana, Chía, Colombia
}

Artículo recibido en abril de 2016, artículo aceptado en octubre de 2016

Citación del artículo: Acuña, L., Cabrera, V., Medina, D., \& Lizarazo, F. (2017). Necesidades de la familia y de la escuela en la educación de niños y niñas con discapacidad intelectual. I+D Revista de Investigaciones, 9 (1), 126 - 137

\begin{abstract}
Resumen
El objetivo de esta investigación fue profundizar en las necesidades de las familias y la escuela en la educación de niños y niñas con discapacidad intelectual, teniendo en cuenta la percepción de padres, profesores, estudiantes con discapacidad y sus compañeros de clase. Para esto se realizó una entrevista semiestructurada a profundidad, que permite conocer detalles significativos de las experiencias.
\end{abstract}

Aunque la educación inclusiva promueve el desarrollo de niños con discapacidad, es importante que padres y profesores cuenten con capacitación para lograr este propósito. De igual forma, es necesario que familia y escuela aúnen estrategias educativas con el fin de contribuir a la formación de los niños con discapacidad en estos dos contextos y así elevar su calidad de vida.

Palabras clave: Familia, escuela, educación, discapacidad intelectual.

\section{Abstract}

The objective of this research was to understand and deepen on the needs of families and schools in establishing social relations of children with intellectual disabilities, taking into account the perception of parents, teachers, children with disabilities and their classmates, survey was depth semi-structured interview was used to find out significant

1. Artículo empírico, enfoque cualitativo, resultado de un proyecto de investigación culminado, perteneciente al Instituto de la Familia, maestría en Asesoría familiar y gestión de programas para la familia, Universidad de La Sabana de la ciudad de Chía, Cundinamarca (Colombia), campus del Puente del Común, km 7, autopista Norte de Bogotá, teléfono: 8615555. Fecha de inicio: 2013, fecha de terminación: 2015

2. Magister en Asesoría familiar y gestión de programas para la familia, Universidad de La Sabana. Instituto de la Familia, Universidad de La Sabana (Colombia). Dirección: Campus del Puente del Común km 7, autopista Norte de Bogotá. lina.acuna@unisabana.edu.co.

3. Doctoranda en Educación, Universidad de Navarra, Magister en Psicología Universidad de Los Andes, Coordinadora de investigación del Instituto de la Familia Universidad de La Sabana (Colombia). Dirección: Campus del Puente del Común, km 7, autopista Norte de Bogotá. Correo institucional: victoria.cabrera@unisabana.edu.co.

4. Licenciada en Educación con énfasis en educación especial, Universidad Pedagógica Nacional. Especialista en Procesos de lecto-escritura, Universidad Minuto de Dios, estudiante de maestría en Asesoría familiar y gestión de programas para la familia, Universidad de La Sabana (Colombia). Correo institucional: dianamedca@unisabana.edu.co

5. Licenciada en Educación básica con énfasis en humanidades, lengua castellana y matemáticas, Universidad Pedagógica y Tecnológica de Colombia. Especialista en Ética y Pedagogía, Fundación Universitaria Juan de Castellanos, estudiante de maestría en Asesoría familiar y gestión de programas para la familia, Universidad de La Sabana (Colombia).Correo institucional: florlisa@unisabana.edu.co 
details of the experiences.

While it is true that inclusive education promotes the development of children with disabilities it is important that parents and teachers count on training to achieve this purpose. Similarly families and school should join forces to find educational strategies in order to contribute to the education of children with disabilities in both contexts and thus raise their quality of life.

Key words: Family, school, education, intellectual disabilities.

La historia de las personas con discapacidad ha estado marcada por las luchas para conseguir igualdad, porque sus derechos sean respetados y se les reconozca no solo desde sus limitaciones sino desde sus diferentes capacidades y habilidades.

Munuera (2013) presenta el transcurrir histórico de las personas en condición de discapacidad explicando que

(...) hasta llegar a la situación actual se ha recorrido un camino de sufrimiento, discriminación y exclusión social, la historia pone de manifiesto las dificultades de ser considerados ciudadanos con los mismos derechos que el resto, los cambios producidos en la sociedad en el tratamiento de estas personas han sido fruto de la lucha de organizaciones sociales formadas por personas afectadas y sus familiares, logrando derechos civiles para su equiparación social. (p. 164)

Como lo explican Moreno y Rojas (2013), una de las situaciones encontradas es la exclusión de las personas con discapacidad de las dinámicas cotidianas en los diferentes contextos en los que participan: familia, estudio y trabajo, entre otros, así como carencia en los ajustes educativos necesarios, lo que acentúa la exclusión.

La escuela, siendo un contexto fundamental para el aprendizaje de las personas con discapacidad, tiene necesidades relacionadas con la importancia del conocimiento y reconocimiento de los otros y con estrategias para brindar una óptima educación. Hegarty (1994) afirma que:

Un objetivo principal de los países debiera consistir en asegurarse que docentes en cursos de capacitación aprendan algo acerca de las discapacidades y de sus implicancias educacionales. No se trata que se conviertan en expertos, pero deberían aprender cómo modificar el contenido curricular y el enfoque docente de las aulas. También, adquirir destrezas en identificación y evaluación de estudiantes con discapacidades, apreciar la importancia de trabajar con padres y desarrollar destrezas apropiadas. (p. 26)
Es fundamental que entre estos dos contextos exista una cooperación que facilite permanente conexión y equilibrio para mejores resultados, ya que "(...) los alumnos rinden más cuando padres y profesores comprenden sus expectativas mutuas y se mantienen en contacto" (Redding, 2002, p. 21). De acuerdo con los resultados, es necesario reflexionar sobre la importancia de que instituciones especializadas y responsables de la educación atiendan con prioridad las necesidades de familia y escuela.

Con este estudio se pretende aportar en el mejoramiento de las condiciones de vida familiar y de educación de estos niños, debido a que las falencias que se están presentando son resultado de falta de conocimiento, formación, estrategias y procesos asertivos en su educación.

Es importante reconocer tanto las funciones de la familia como las de la escuela, y la relevancia del trabajo de ambas en el desarrollo de los niños y las niñas (León, 2011) "(...) la escuela fue creada para favorecer el desarrollo de los niños y niñas y adelantar procesos propios de escolaridad, y para esto es necesario implicar a la familia en el proceso, siendo consciente del rol que desempeña" (p. 16).

El Estado colombiano ha realizado un esfuerzo importante con el fin de reconocer la dignidad de las personas con discapacidad, por lo que desde la Constitución Política de 1991 son reconocidos sus derechos, haciendo referencia a la responsabilidad del Estado en protección, atención, apoyo e integración social de personas con esta condición. Con respecto a la educación, la Ley 115 de 1994, Ley General de Educación, en el Capítulo 1, Título III, artículos 46 a 49 hace referencia al apoyo que el Estado debe ofrecer para fomentar programas y experiencias que permitan una adecuada atención educativa a personas con limitaciones o capacidades excepcionales (Consejería Presidencial para la Política Social [Conpes], 2002).

Igualmente, las necesidades de las familias de niños en condición de discapacidad están contempladas en la Convención de la Organización de las Naciones 
Unidas ([ONU], 2006) sobre los derechos de personas con discapacidad; en esta se evidencia que tanto la persona en condición de discapacidad como su familia deben recibir una atención especial, teniendo en cuenta que "(...) el desarrollo de las personas en condición de discapacidad intelectual depende de los mismos factores que son importantes para las personas sin ninguna discapacidad". (Gràcia \& Vilaseca, 2008, p. 47)

La sociedad en general reconoce el papel de la familia y su influencia tanto en la evolución como en el desarrollo de quienes la componen:

La familia es el primer contexto socializador por excelencia, el primer entorno donde miembros que la forman evolucionan y desarrollan a nivel afectivo, físico, intelectual y social. La familia con un hijo con necesidades especiales debe desempeñar las mismas funciones que las demás, la diferencia está en que es más difícil de cumplir cuando se trata de atender al hijo con discapacidad, pues recursos y apoyos de todo tipo se hacen más necesarios y, en ocasiones, permanentes y, en la mayoría de los casos, las familias no están preparadas para dar respuesta a funciones derivadas de las mismas. (Sarto, 2001, párr. 3)

La familia, tradicionalmente, ha sido la encargada de la formación desde pequeños, y sobre ella recae la mayor responsabilidad de satisfacer todas las necesidades de los niños, pero no es hasta finales del siglo XIX, con la industrialización, hasta cuando se empieza a observar que existen muchos beneficios para los niños, que los miembros de la familia estén juntos para enseñarles, entre otras cosas, valores sociales y prepararlos para la incorporación al mundo. La escuela se convierte así, lentamente, en otro contexto de desarrollo para la infancia, fortaleciendo con esto el planteamiento de las relaciones que deben existir entre familia y escuela. (García, 2003; Cagigal, 2003)

En cuanto a la discapacidad intelectual, la Asociación Americana de Discapacidad del Desarrollo ([AAIDD], 2010) la ha definido como una condición "(...) caracterizada por limitaciones significativas en el funcionamiento intelectual y en la conducta adaptativa que se manifiesta en habilidades adaptativas conceptuales sociales y prácticas". Quienes tienen esta condición presentan una disminución en la habilidad de entender información nueva o compleja, así como dificultad para aprender a aplicar nuevas habilidades; esto se evidencia en que son personas con un menor grado de independencia y dificultades en el funcionamiento social. (Shogren et al., 2010)
Por su parte, Verdugo (1994) ha desarrollado un nuevo concepto en el que se incluye la interacción de la persona con discapacidad con su propio contexto, así como una visión multifactorial de la etiología y la diferenciación entre una definición operativa y otra constitutiva de la condición de discapacidad (Verdugo \& Shalock, 2010), características que son importantes para explicar las limitaciones dentro de un contexto social.

De acuerdo con lo anterior, esta investigación pretendió analizar la comprensión de las necesidades de educación de niños y niñas con discapacidad intelectual en dos contextos significativos como son la familia y la escuela, de acuerdo con la percepción de las personas que interactúan con estos niños.

\section{Método}

\section{Tipo de estudio}

Se trata de un estudio transversal descriptivo de investigación social cualitativo "(...) que se refiere en su más amplio sentido a la investigación que produce datos descriptivos: las propias palabras de personas, habladas o escritas, y la conducta observable" (Taylor \& Bogdan, 2000, p. 7), con el fin de obtener la percepción de padres, madres, cuidadores, profesores y pares con discapacidad cognitiva en aspectos relacionados con la educación. Las narrativas de los entrevistados se analizaron a través del análisis del discurso, de acuerdo con los postulados de Potter y Wetherell (1987), con el fin de conocer los repertorios interpretativos de los participantes relacionados con asuntos de la educación de los niños con discapacidad.

La información se recolectó a través de entrevistas semiestructuradas a profundidad, teniendo en cuenta que

(...) la intencionalidad principal de este tipo de técnica es adentrarse en la vida del otro, penetrar y detallar lo trascendente, descifrar y comprender gustos, miedos, satisfacciones, angustias, zozobras y alegrías, significativas del entrevistado; consiste en construir paso a paso y minuciosamente la experiencia del otro. (Robles, 2011, p. 40)

Para el diseño de las preguntas de la entrevista se tuvo en cuenta el cuestionario denominado"Escala de evaluación de necesidades familiares de familias de personas con discapacidad intelectual y del desarrollo" que validaron Chiu, Turnbull y Summers (2013). Ver Anexo 1. 


\section{Participantes}

Se incluyeron en el estudio veintiún participantes pertenecientes a una institución educativa con estudiantes en condición de discapacidad. Las características de los participantes se muestran en la Tabla 1.

\section{Tabla 1}

\section{Características de los participantes}

\begin{tabular}{|c|c|c|c|}
\hline & Masculino & Femenino & \\
\hline $\begin{array}{l}\text { Alumnos con } \\
\text { discapacidad cognitiva }\end{array}$ & 5 & 1 & 9-12 años \\
\hline Compañeros de estudio & 1 & 4 & \\
\hline Profesores & 1 & 2 & \\
\hline Padres & & 2 & \\
\hline Madres & & 4 & \\
\hline Madrastra & & 1 & \\
\hline
\end{tabular}

\section{Fuente: Los Autores}

\section{Procedimiento}

En la primera fase se invitó a los padres de todos los estudiantes en condición de discapacidad intelectual y se les explicaron los objetivos y la forma de participación, y se acordó una fecha para realizar las entrevistas a aquellos que voluntariamente quisieran hacer parte de la investigación. Se explicó que la participación era anónima y que toda la información se manejaría de forma confidencial, solo con fines investigativos.

En la segunda fase, una vez se confirmaron los participantes, se realizó la firma del consentimiento informado y se procedió a hacer las entrevistas a padres e hijos con discapacidad intelectual.

Posteriormente, fueron contactados los profesores, con quienes se siguió el mismo procedimiento, y finalmente, a los padres de los compañeros de clase de los alumnos en condición de discapacidad, para que firmaran el consentimiento para que sus hijos pudieran participar del estudio.

\section{Resultados}

Esta investigación se acercó a las necesidades de educación de dos contextos significativos para las personas con discapacidad intelectual, la familia y la escuela. Una vez analizada la información recogida de cada grupo de participantes se identificaron las necesidades más relevantes para cada uno de ellos en el rol y contexto en el que se desenvuelve. Ver Figura 1.

Gráfico 1. Figura de análisis por categorías de los resultados.

Gráfico 1. Figura de análisis por categorías de los resultados.

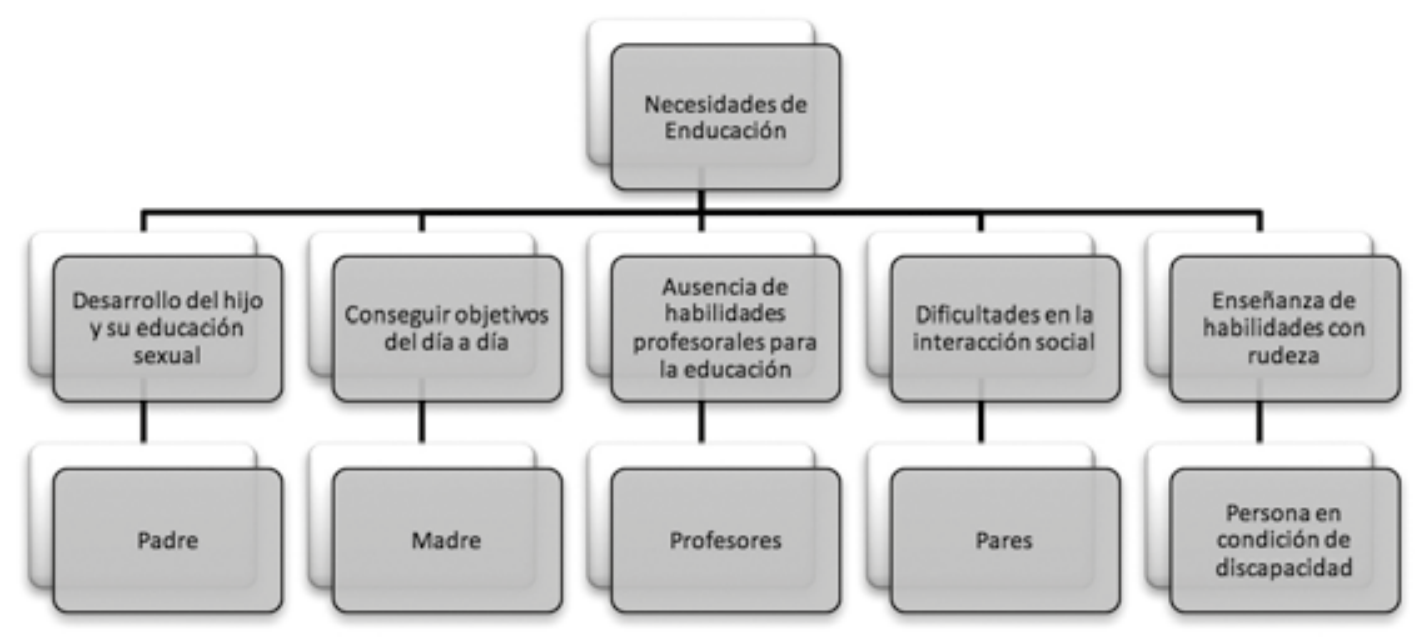

Figura 1.

Figura de análisis porcategorías de los resultados. Fuente: Autores. 
Desarrollo del hijo y su educación sexual. Percepción paterna.

Una de las preocupaciones de los padres es el momento del ciclo vital del hijo con discapacidad intelectual, específicamente lo relacionado con los cambios propios de la adolescencia. Ejemplo de ellos son algunos comentarios que hacen los padres: "Yo quería que él siguiera siendo bebé"; "Ya no le gusta que le dé picos"; "Papi, picos no", "Eso siempre me pelea por los picos". Por otro lado, como ocurre con hijos sin esta condición, existe el temor acerca de la manera de abordar temas de sexualidad y de las relaciones con el sexo opuesto: "Él me pregunta cosas, cosas que yo no le puedo decir, por ejemplo, esa atracción por lo femenino que a él se le ha despertado, de tener a alguien que de pronto le diga "hola, papito, hola, esto". Yo le digo 'hola, papito, hola, mi amor', pero a él ahora le atrae que alguien del sexo opuesto se lo diga".

En ese mismo sentido, se evidencia dificultad en el abordaje de los temas relacionados con la sexualidad. Cuando se indagó por la formación en este tópico, se obtuvo lo siguiente: "Ehhhh, bueno, ahí sí (risas) lo que se dice hablar de sexualidad, no, no, no, pero es un tema que tengo que abordar". Sin embargo, es necesario educar al hijo en la dimensión sexual, aunque al padre (madre) de familia no se lo hayan enseñado, pero aún más relevante es hacerlo cuando el padre o la madre cuente con los conocimientos del tema para enseñarlo: "A mí no me lo enseñaron de pequeño, pero uno tiene que enseñarlo, y uno ya sabiéndolo, lo tiene que enseñar."

La dificultad del padre para hablar de la educación de la sexualidad y temas relacionados con esta, se debe a que él también carece de formación al respecto. Por tal razón, es necesario que se busquen estrategias para que a los padres de las personas con discapacidad intelectual se les acompañe y guíe en esta formación, y se les proporcionen orientaciones pertinentes para que la educación de la sexualidad de los hijos con discapacidad intelectual no siga siendo una necesidad, sino una fortaleza de la familia (Acuña, Cabrera \& Suárez, 2016).

\section{Conseguir los objetivos del día a día. Percepción materna}

Tener un miembro en la familia con discapacidad, es, en la mayoría de los casos, una situación difícil, que se hace más compleja cuando no se tiene conocimiento de las características propias de la discapacidad y de la manera como la familia puede aportar a la educación y el desarrollo óptimo de la persona con discapacidad. Una madre dice: "Quisiera saber cuál es la estrategia para que mi hijo haga a la primera vez lo que le pido, y no tener que repetírselo varias veces".
La madre expresa el vacío que siente en aspectos importantes como la forma en que aprende su hijo con discapacidad intelectual, y el desconocimiento de estrategias que puede emplear para que sus aprendizajes sean significativos. En consecuencia, es preciso que se aborde esta necesidad educativa, ofreciendo a los padres una formación que les facilite un mejor trabajo como familia, que permitirá, además, fortalecer las relaciones entre madre e hijo: "Yo sí tengo duda de cómo debe uno manejar esto, y por eso quisiera que me dieran algunas pautas para sobrellevarlo". Emocionalmente me siento..., no sé qué hacer, él poco comparte conmigo, llega del colegio, tira la maleta y se va para donde el amiguito".

La madre busca herramientas para comprender y aceptar la condición de su hijo, a fin de poder entender cuáles son sus necesidades y cómo ayudarle, en este caso necesita entender cómo aprende su hijo, para así poder enseñarle de la forma más acertada. Por ello, las estrategias que utilice la familia para que las personas con discapacidad intelectual hagan lo que se les pide, deben estar mediadas por la comprensión de su condición, de sus habilidades y lo que les falta por aprender: "Yo quisiera que él despegará por sí solo, que él hiciera las cosas por él mismo, pero no sé qué pasa con él, en la casa me toca amenazarlo con la correa, entonces no sé".

\section{Ausencia de habilidades profesorales para la educación. Percepción del profesorado}

En el derecho a la educación es importante reflexionar sobre las habilidades de los profesores que hacen parte de la escuela inclusiva, ya que es pertinente que cada uno de ellos reciba una formación adaptada a las necesidades de educación de los niños con discapacidad. Por ello, se debe tener en cuenta que los profesores requieren de una formación idónea para educar a niños de diversas condiciones intelectuales, culturales y familiares. No obstante, lo cierto es que la formación de profesores en este sentido, es una de las necesidades de la escuela, pues algunos docentes no se sienten preparados para dar respuesta a las diversas necesidades de los niños: "No me siento preparado para atender a niños con discapacidad simultáneamente con los que no tienen discapacidad, a uno no lo forman para esto".

La competencia profesional del docente que tiene a cargo la educación de niños con discapacidad intelectual es un requerimiento esencial para hablar de educación de calidad, y los profesores deben contar con conocimientos que les permitan atender las necesidades de estos niños. Como lo menciona uno de ellos: "Yo creo que es importante que el colegio sepa cómo se debe tratar a los niños de inclusión, eso me ayudaría mucho, porque uno 
ya sabría qué hacer, como que uno no puede encarrilarlos igual que a los otros chicos, con este niño el trabajo es diferente, el trato es diferente, lo mismo en el salón, no los puedo mover de adelante".

El trabajo que debe hacer el profesor en una escuela inclusiva es fundamental, por lo tanto, hay que comprender su quehacer, pero sobre todo, que las instituciones encargadas de la educación satisfagan sus necesidades, como es su formación: "Si yo sé cómo es el niño, eso ayuda en la forma de enseñarle, en la forma de comprenderlo, pudo decir 'ya sé por qué el niño es así, ya sé qué estrategia de pronto usar con él,' y hasta tenerle más paciencia".

\section{Dificultades en la interacción social. Percepción de los pares}

La escuela es un lugar de encuentros que se deben encarar con respeto y tolerancia por la condición del otro. Por ello, aunque diferentes, los estudiantes deben asumir responsabilidades y respetar normas, por lo que es importante que todos, incluyendo las personas con discapacidad, trabajen por mantener un ambiente saludable en pro de mejores relaciones y una mejor convivencia. Una compañera de curso de un estudiante con discapacidad dijo: "Él siempre es desjuiciado y arregla los problemas dando puños, en el descanso se porta mal, le falta aprender a comportarse. Yo no soy amiga de él porque él me pega".

En este sentido, es fundamental que a todos los estudiantes que hacen parte de la escuela inclusiva cuenten con espacios de aprendizaje en medio de los conflictos diarios, que les permitan convivir y tolerar las diferencias del otro, desde lo que comparten hasta lo que los separa, basados en el respeto y el cuidado del otro: "Él es peligroso, un día casi me tira del balcón, una amiga mía me defendió"; "Algunos niños le tienen fastidio porque dicen que él es como raro y también muy feo, ah, y muy molestón".

Por lo tanto, escuela y familia deben trabajar en la formación no solo de conocimientos, sino también, de habilidades y destrezas que permitan al estudiante relacionarse con los demás en cualquier contexto: "Él vive en una esquina y yo en la otra, cuando él le va hacer un mandado a la mamá va hasta la tienda y pasa al lado de mi casa, y mi mamá también me da plata para comprar algo, y cuando yo ya estoy lista para ir a la tienda. No quiero encontrármelo porque él siempre me molesta, aun cuando mi mamá esté conmigo él a mí me molesta".

\section{Enseñanza de habilidades con rudeza. Percepción de los hijos en condición de discapacidad}

Las dinámicas que deben atravesar tanto las familias con un hijo con discapacidad como la persona que lo cuida, son diversas, entre estas se encuentra el tiempo en familia y las necesidades económicas. Una persona con discapacidad requiere de una mayor demanda de atención y tiempo, así como una serie de gastos adicionales. Un hijo con discapacidad intelectual expresó: "Ella (mamá) me lo enseña a gritos; no me gusta cuando mi mamá me dice con gritos lo que tengo que hacer, me gustaría que me lo enseñara diferente, pero primero tiene que darse un descanso de las empanadas y eso, para que ella se relaje, pero ella siempre tiene que hacer empanadas, empanadas y empanadas, ¿qué importa más, el dinero o la familia? La familia".

Los padres deben afrontar situaciones propias de la vida cotidiana, como la conciliación que deben hacer entre el tiempo en familia y el trabajo. Esta conciliación es un indicador de la calidad de vida familiar. Tales situaciones se pueden equilibrar: "Mi mamá no me pone cuidado porque se la pasa trabajando, cuando yo le digo que ella (hermana) me pega y me dice groserías".

Para el bienestar emocional de los hijos con discapacidad es importante que los padres tengan en cuenta que sus acciones y comportamientos afectan a sus hijos, y deben saber gestionar sus emociones, angustias y miedos, y buscar otras posibilidades de enseñanza diferente a los gritos en el proceso educativo de sus hijos: "Yo no aprendo a los gritos, yo aprendo leyendo, con los gritos me siento mal".

Conocer a las personas con las que se comparten distintos momentos, como en la escuela, es fundamental para comprenderlas mejor. Es importante que a los integrantes de la comunidad educativa se les haya sensibilizado, capacitado y acompañado en la comprensión de las características propias de las personas con discapacidad, pues como lo menciona un estudiante con discapacidad intelectual, el concepto que se tiene de él no es el más apropiado: "Algunos compañeros piensan que soy raro, anveces (sic), cuando hablo, no me dicen nada y se van, solo una vez no me dicen nada y lo dejan así."

Es fundamental que para mantener buenas relaciones en la escuela inclusiva se reconozca a los otros y se entiendan sus diferencias: "Me han dicho pirata, bobo, porque no sabían lo que tenía". El niño con discapacidad se siente mal cuando los otros niños del colegio los atacan, y se sienten mal por su condición de vulnerabilidad: "Hay un niño que me pega, y cuando nos vamos a ir nos 
persigue para pegarnos con una piedra"; "A cada rato me molestan, un niño le botó el balón a otro y me echó la culpa a mí, ahora me toca pagar el balón"; "Me ofenden cuando me dicen groserías".

\section{Discusión}

El objetivo de esta investigación fue profundizar en las necesidades de las familias y la escuela en la educación de los niños con discapacidad intelectual, teniendo en cuenta la percepción de padres, profesores, estudiantes con discapacidad y compañeros de clase, con el fin de aportar en el mejoramiento de las condiciones de vida familiar y de educación de esta población.

\section{Necesidades de la familia en la educación de los niños con discapacidad intelectual}

En la familia existen diversas necesidades relacionadas con la falta de conocimiento sobre diversos temas; en el caso del padre, se relaciona con la carencia de herramientas para hablar con su hijo de sexualidad, y en el de la madre, conocer mejor sobre la discapacidad de su hijo y así comprender sus comportamientos y ofrecerle el apoyo pertinente. Dicho desconocimiento tanto del padre como de la madre en la educación del hijo, lleva a reflexionar sobre la necesidad de capacitar a los progenitores y cuidadores de los niños con discapacidad sobre la forma en que deben tratarlos y educarlos, siempre respetando su condición intelectual. Esto va en línea con lo que explica Muñoz:

(...) la información es un derecho de los padres que ayuda a comprender muchos comportamientos de su hijo/a, que en ocasiones, pueden generar angustia por carecer de justificación. La información que se da a las familias sobre el tipo de discapacidad y sobre métodos de actuación es de alta importancia, pues mediante ésta, se favorece el desarrollo integral de su hijo/a. Esto facilita el conocimiento del niño/a en profundidad, su potencial, sus puntos fuertes $y$ débiles para desarrollarlos. $(2011$, p. 5)

Enseñarles a los hijos con discapacidad sobre sexualidad y educarlos en esta dimensión es un asunto complejo, por ello, es necesario que los padres primero conozcan a sus hijos, que tengan una relación cercana con ellos. Esto se asocia con lo que menciona Davis y Modell, en cuanto a que:

... es imperativo y fundamental que a los padres se les acompañe en este proceso de aprender, conocer y comprender primero sobre la discapacidad de sus hijos, y así, la manera como ellos pueden abordar temas relacionados con la sexualidad, y otros temas en los que también se deben educar. (s. f, p. 4)

Las falencias que reporta el hijo con discapacidad se refieren a la manera como su madre le habla, la forma de enseñarle mediante los gritos y el poco tiempo para compartir con él, debido a las ocupaciones laborales. La falta de conocimiento de los padres sobre las condiciones de sus hijos los llevan a manejar de manera inadecuada las situaciones conflictivas y estresantes que se presentan en la familia. Fantova (2000, p. 11) explica que estas situaciones estresantes pueden poner en crisis a la persona (padres) desde lo emocional, lo cognitivo y lo comportamental, y de esta manera afectar, no solo la relación con los hijos, sino consigo mismos y con el sistema familiar en general.

Es claro que la familia tiene varias necesidades, pero también es claro que su trabajo, funciones y objetivos para con las personas con discapacidad intelectual son esenciales, pues su acompañamiento y guía influyen notoriamente en el desarrollo de estas personas. Pozzi y Valdés $(2008$, p. 8) priorizan la importancia de la familia entendiendo que:

(...) allí es donde se expresan acciones cotidianas, sentimientos, emociones e ideas. Es el entorno donde niños y niñas aprenden por primera vez formas básicas de convivencia social: estilos de comunicación, expresión de sentimientos, comprensión, buenos hábitos, sentido de la solidaridad y colaboración para el logro del bien común y, ante todo, el respeto por sí mismo y por los demás. En pocas palabras, en familia se aprende a compartir lo que se es, lo que se tiene y lo que se hace.

\section{Necesidades de la escuela en la educación de niños y niñas con discapacidad intelectual}

En el contexto escolar se destaca la importancia de las habilidades del profesor en la educación de los niños con discapacidad intelectual, su preparación, así como la promoción de una sana convivencia entre los estudiantes, que parte de la diversidad de la escuela inclusiva y la importancia de comprender al otro.

El derecho a la educación es fundamental y se debe garantizar, pero para este fin es necesario también tener en cuenta la necesidad de formación de los profesores, quienes perciben esta educación como una prioridad para atender a las diferentes poblaciones en una escuela inclusiva. De acuerdo con Durán y Giné (2011), la educación inclusiva se debe entender como un proceso 
de formación y de capacitación del profesorado y de los sistemas educativos en general, que busca atender el alumnado en medio de la diversidad.

En este sentido, también surge la necesidad de acompañar a todos los integrantes de la comunidad educativa en el acercamiento y reconocimiento de las personas con discapacidad intelectual, para comprender sus particularidades, ya que como lo afirma García (2011), estas personas deben lidiar con más barreras a la hora de integrarse, en ocasiones por el miedo o el desconocimiento que, muchas veces, las personas tienen para relacionarse con ellos.

Las personas con discapacidad intelectual, como parte de una escuela inclusiva, deben -al igual que todoseducarse para mantener buenas relaciones con los demás y una sana convivencia; su condición no los exime de respetar y cumplir normas.

Este resultado está en la misma línea de lo propuesto por Barrio (2009) quien explica que debe haber normas y reglas de comportamiento claras para todos, producto de un consenso, al igual que normas relacionadas con la resolución de conflictos. Amaro (2014) explica que la sociedad actual exige un tipo de ciudadano que posea conocimientos, habilidades y destrezas, que sea competente en resolución de problemas y útil en el trabajo que desempeñe, sin embargo, debido a la crisis moral que se observa en la actualidad, también exige que posea valores no sólo para acatar y respetar leyes y normas, sino valores que permitan resolver conflictos en los ámbitos de su vida y convivir armónicamente en el grupo al que pertenezca.

Es de destacar, entonces, que en el contexto escolar el respeto por la diversidad debe ir orientado hacia la importancia de comprender, aceptar y valorar a otros desde sus particularidades. Parra afirma que

(...) la educación debe propender por la construcción del conocimiento individual a partir de incorporación e internalización de pautas culturales, de compartir conocimientos, y se constituye en la base necesaria para el aprendizaje. De este modo, al tener en cuenta la inclusión desde el punto de vista educativo, se entiende que es hacer efectivo para todos el derecho a la educación, que contempla la igualdad en las oportunidades, la eliminación de barreras para el aprendizaje y la participación en el contexto físico y social. (2011, p. 147)

\section{Conclusiones}

Todos los niños necesitan recibir de sus padres, desde temprana edad, formación en temas relacionados con sexualidad. Si bien la familia es consciente de la importancia del tema y se ha ido aproximando a la enseñanza de esta dimensión humana, se muestra temerosa ante la inminencia de tener que abordarla en la etapa de la adolescencia, por no contar con herramientas educativas para hacerlo, más que por falta de voluntad.

Las estrategias que los padres utilizan para enseñarles a sus hijos están condicionadas por la poca formación que tienen, evidenciando una necesidad de apoyo sobre otras formas de enseñar y descubrir los talentos de sus hijos para garantizar no solo la adquisición del aprendizaje sino que este ocurra de manera agradable, siendo importante en cualquier tipo de enseñanza el conocimiento de las características propias de la persona con discapacidad intelectual.

Se debe apoyar la familia en la consolidación de procesos que eduquen a cada uno de sus miembros, y la mejor manera de hacerlo es trabajando en la comprensión e importancia de fortalecer los lazos familiares, pues si bien es cierto que el tiempo es fundamental para mantener la unión familiar, el aspecto económico también lo es, en tanto que parte importante para tener una mejor calidad de vida y dar respuestas a muchas de las necesidades.

Los estudiantes en condición de discapacidad intelectual necesitan que la escuela les brinde una educación, además de pensada en sus capacidades cognitivas, enfocada en su condición de seres humanos que se relacionan con otros y con el entorno.

Para que los estudiantes en condición de discapacidad desarrollen sus potencialidades académicas y relacionales en el entorno escolar necesitan un currículo flexible que conduzca hacia el desarrollo de todas sus capacidades. De igual forma, escuelas que junto con sus maestros y demás integrantes de la comunidad educativa promuevan el respeto hacia la diversidad y corresponsabilidad entre todos los actores involucrados en la educación.

Para obtener mejores resultados en una escuela inclusiva sedeben tener en cuenta las necesidades delos profesores y la gran responsabilidad que tienen en la educación de todos los niños y niñas con o sin discapacidad. Por tal razón, se les debe capacitar con el fin de garantizar las herramientas necesarias para que puedan llevar a cabo, con calidad, la formación y atención de esta población. 
La escuela debe ser un espacio que acoja a la familia, reconociendo la labor de enseñanza que desarrolla en su interior, teniendo en cuenta sus fortalezas y debilidades pero también sus potencialidades, de tal manera que las dos se consideran entre sí como instituciones recíprocas y complementarias en la tarea de la formación de los educandos.

Es necesario poder replicar estudios de este tipo en otros entornos, con el fin de obtener resultados reproducibles con resultados válidos producto de investigaciones cualitativas, como lo expresan Sánchez y Córdoba (2014).

\section{Agradecimientos}

El grupo de investigación que elaboró este artículo agradece a la Universidad de La Sabana el apoyo brindado en el desarrollo de la investigación en general. Asimismo, a las personas con discapacidad intelectual y sus familias, sus cuidadores y miembros del contexto escolar que facilitaron el acceso a la información.

\section{Referencias}

Acuña, L., Cabrera, V. \& Suárez. A. (2016). Necesidades en la educación de hijos e hijas con discapacidad intelectual según la percepción de sus cuidadores. Revista Criterios, 23(1), 289-306.

Amaro, A. (2014). La integración de alumnos con necesidades educativas especiales a la escuela regular como parte de los principios de diversidad e igualdad en el desarrollo de los futuros ciudadanos. Revista de educación, cooperación y bienestar social, 3, 73-77. Recuperado de http://www. revistadecooperacion.com/numero3/03-05.pdf

Barrio, J. (2009). Hacia una educación inclusiva para todos. Revista Complutense de Educación, 20, 13-31. Recuperado de https://revistas.ucm.es/index.php/RCED/article/ viewFile/RCED0909120013A/15360

Cagigal, V. (2003). La relación familia-escuela: un entramado de crecimiento hoy. Conversaciones pedagógicas, 1-23. Recuperado de http://webs.uvigo.es/consumoetico/ carmenpereirappersonal.htm\#actividades_academicas.

Chiu, C., Turnbull, A. \& Summers, J. (2013). What families need: Validation of the Family Needs Assessment for Taiwanese families of children with intellectual disability and developmental delay. Research and Practice for Persons with Severe Disabilities, 38(4), 247-258.

Consejería Presidencial para la Política Social. (2002). Plan nacional de atención a las personas con discapacidad.
Manual operativo. Recuperado de http://discapacidad.8m. net/MANUAL.htm

Davis, M.\&Modell, S. (s.f.). Protegiendo niños con discapacidades del asalto sexual. Guía para padres. Recuperado de http:// kc.vanderbilt.edu/kennedy_files/ bilitiesfromSexualAssaultGuideEspanol.pdf

León De, B. (2011). La relación familia-escuela y su repercusión en la autonomía y responsabilidad de los niños/as. XII Congreso internacional de teoría de educación. Universidad de Barcelona, España. Recuperado de https://extension.uned.es/archivos_publicos/webex actividades/5385/repercusiones8.pd

Durán, D. \& Giné, C. (2011). La formación del profesorado para la educación inclusiva: Un proceso de desarrollo profesional y de mejora de los centros para atender la diversidad. Revista Latinoamericana de Inclusión Educativa, 5, 153170. Recuperado de http://www.rinace.net/rlei/numeros/ vol5-num2/art8.html

Fantova, F. (2000). Trabajar con las familias de las personas con discapacidades. Siglo cero, 31, 33-49. Recuperado de http://dialnet.unirioja.es/servlet/revista?codigo $=1348$

García, F. (2003). Las relaciones escuela-familia: un reto educativo. Infancia y aprendizaje, 4, 425-437. Recuperado de http://nx.educacionenmalaga.es/valores/ files/2011/12/Relaciones-escuela-familia1.pdf

García, M. (2011). Habilidades sociales en niños y niñas con discapacidad intelectual. Recuperado de http://www. eduinnova.es/monografias2011/ene2011/habilidades.pdf

Grácia, M. \& Vilaseca, R. (2008). Cómo mejorar la calidad de vida de las familias de niños con discapacidad intelectual: algunas propuestas. Revista Española sobre discapacidad intelectual, 39, 44-62. Recuperado de http://sid.usal.es/ idocs/F8/ART11908/calidad_vida_familias.pdf

Hegarty, S. (1994). Educación de niños y jóvenes con discapacidades principios y práctica. Unesco. Recuperado de http://www.unesco.org/education/pdf/281_65_s.pdf

Munuera, M. (2013). Mediación con personas con discapacidad: igualdad de oportunidades y accesibilidad de la justicia. Política y sociedad, 1, 163-178. Recuperado de https:// www.academia.edu/6808301/Mediaci\%C3\%B3n_ con_personas_con_discapacidad_igualdad_de_ oportunidades_y_accesibilidad_de_la_justicia

Moreno, D. \& Rojas, V. (2013). Diseño incluyente desde el proceso comunicativo usuario-dispositivos para divergencia 
funcional locomotriz. I+D Revista de Investigaciones, 1(1), 61-68.

Muñoz, A. (2011). Impacto de la discapacidad en las familias. Innovación y experiencias educativas, 38, 1-8. Recuperado de http://www.csi-csif.es/andalucia/modules/mod_ense/ revista/pdf/Numero_38/ANASTASIA_MUNOZ_1.pdf

Organización de las Naciones Unidas. (2006). Convención Internacional sobre los Derechos de las Personas con Discapacidad y Protocolo Facultativo. Recuperado de http://www.un.org/disabilities/documents/convention/ convoptprot-s.pdf

Parra, C. (enero-junio, 2011). Educación inclusiva: un modelo de diversidad humana. Revista educación y desarrollo social, 5, 139-150. Recuperado de http://www.umng.edu.co/ documents/63968/76126/10.pdf

Potter, J. \&Wetherell, M. (1987). Discourse and social psychology. London: Sage.

Pozzi, E. \& Valdés, R. (2008). Ayudemos al niño a aprender a convivir. Guía para el cuidado y desarrollo del niño de 6 a 12 años. Recuperado de http://www.unicef.org/uruguay/ spanish/Guia_maestros_3_ultima.pdf

Redding, S. (2002). Familias y escuelas. Series prácticas educativas, 2, 1-39. Recuperado de https://extension. uned.es/archivos_publicos/webex_actividades/5385/ repercusiones4.pdf

Robles, B. (2011). La entrevista en profundidad: Una técnica útil dentro del campo antropofísico. 18, 39-49. Recuperado de http://www.redalyc.org/articulo.oa?id=35124304004
Sánchez, M. \& Córdoba, E. (2014). Proceso de replicabilidad en la educación especial en el Instituto Panameño de Habilitaciòn Especial, 2007-2012. I+D Revista de Investigaciones, 2(1), 6-18.

Sarto, M. (2001). Familia y discapacidad. III congreso "La atención a la diversidad en el sistema educativo". Recuperado de http://campus.usal.es/ inico/actividades/ actasuruguay2001/5.pdf

Schalock, L., Borthwick, S., Bradley, V., Buntix, H., Coulter, D., Craig, M., ... Yeager, H. (2010). Intellectual disability. Definition, Classification, and Systems of Supports (11th. ed.). Washington, D.C.: American Association on Intellectual and Developmental Disabilities.

Shogren, K., Bradley, V., Gómez, S. Yeager, M. \& Schalock, R. (2010). Política pública y mejora de los resultados deseados para las personas con discapacidad intelectual. Revista Siglo Cero, 42(238), 7 -25.

Taylor, S. \& Bogdan, R. (2000). Introducción a los métodos cualitativos de investigación. Recuperado de http:// www.terras.edu.ar/aula/cursos/10/biblio/10TAYLOR-S-JBOGDAN-R-Metodologia-cualitativa.pdf

Verdugo, M. \& Shalock, R. (2010). Últimos avances en el enfoque de y concepción de las personas con discapacidad intelectual. Revista Siglo Cero. Revista española sobre discapacidad intelectual, 41(4), 7-21.

Verdugo, M. (1994). El cambio de paradigma en la concepción del retraso mental: la nueva definición de la AAMR. Siglo cero. Recuperado de http://sid.usal.es/idocs/F8/ART4099/ verdugo_AAMR_92.pdf 


\section{Anexo 1.}

Preguntas de Escala de evaluación de necesidades familiares de familias de personas con discapacidad intelectual y del desarrollo (Factor educación)

17. Ayudar a mi(s) hijo(s) a conseguir los objetivos durante el día a día.

28. Enseñar a tomar decisiones y resolver problemas. Saber cuándo mi(s) hijo(s) está haciendo progresos.

39.

57. Enseñar habilidades de vida independiente (por ejemplo, comer, y vestirse).

69. Enseñar habilidades sociales y emocionales.

72. Enseñar conductas adecuadas.

74. Enseñar habilidades comunicativas.

76. Enseñar a mi(s) hijo(s) aspectos relacionados con la sexualidad.

77. Ayudar con los deberes. 